\title{
Polimorfismos genéticos associados ao metabolismo lipídico envolvidos na fisiopatologia do acidente vascular encefálico isquêmico
}

\section{Genetic polymorphisms associated with lipid metabolism involved in the pathophysiology of ischemic stroke}

\author{
Tamires Flauzino; ; Daniela Frizon Alfieri²; Ana Paula Kallaur³; Elaine Regina \\ Delicato de Almeida ${ }^{4}$; Edna Maria Vissoci Reiche ${ }^{5}$
}

\begin{abstract}
Resumo
O acidente vascular encefálico (AVE) é uma desordem complexa, multifatorial e poligênica decorrente da interação entre componentes genéticos do indivíduo e fatores ambientais. Estudos prévios têm estabelecido a hipertensão arterial, tabagismo, diabetes mellitus, dislipidemia, elevado índice de massa corpórea, distúrbios da coagulação e aumento da idade como fatores de risco preditores de AVE. A dislipidemia tem sido associada à fisiopatologia do AVE isquêmico e polimorfismos genéticos que ocorrem na via metabólica dos lipídeos têm sido propostos como fatores genéticos associados ao AVE isquêmico. O componente genético na causa da dislipidemia tem sido intensamente investigado nos últimos anos. Entre os vários polimorfismos genéticos, os que ocorrem nos genes Apo E, Apo B, LDLR, Apo A-I, Apo C-III, lipase hepática, proteína transferidora de ésteres de colesterol (CETP), lipase lipoprotéica (LPL) e proteína convertase subtilisina/kexina tipo 9 (PCSK9) têm sido objeto de estudos na população em geral. Os dados sobre o perfil lipídico e o estudo dos polimorfismos dos genes que codificam proteínas estruturais e enzimas relacionadas com o metabolismo dos lipídeos podem revelar a prevalência das dislipidemias em uma população, possibilitando uma intervenção direcionada para o controle e prevenção das doenças ateroscleróticas como o AVE isquêmico.
\end{abstract}

Palavras-chave: Acidente vascular encefálico. Polimorfismo genético. Dislipidemia. Receptor de lipoproteína de baixa densidade.

\begin{abstract}
The stroke is a complex, multifactorial, and polygenic disorder that results from the interaction between the individual genetic components and environmental factors. Previous studies have established hypertension, smoking, diabetes mellitus, dyslipidemia, elevated body mass index, disturbances of coagulation and increasing age as predictors of stroke risk factors. Dyslipidemia has been associated with pathophysiology of ischemic stroke and genetic polymorphisms that occur in the metabolic pathway, such as lipids metabolism, has been one of the hereditary factors related to ischemic stroke. The genetic component in the cause of dyslipidemia has been intensively investigated in recent years. Among the
\end{abstract}

\footnotetext{
${ }^{1}$ Farmacêutica. Mestranda no Programa de Pós-graduação em Ciências da Saúde da Universidade Estadual de Londrina. E-mail: t.flauzino@hotmail.com

${ }^{2}$ Farmacêutica. Mestranda no Programa de Pós-graduação em Ciências da Saúde da Universidade Estadual de Londrina. E-mail: frizon.alfieri@gmail.com

${ }^{3}$ Farmacêutica. Doutoranda no Programa de Pós-graduação em Ciências da Saúde da Universidade Estadual de Londrina. E-mail: anapaulakallaur@yahoo.com.br

${ }^{4}$ Farmacêutica. Doutora em Patologia Experimental. Docente do Departamento de Patologia, Análises Clínicas e Toxicológicas do Centro de Ciências da Saúde da Universidade Estadual de Londrina. E-mail:elainedelicato@hotmail.com

${ }^{5}$ Farmacêutica. Doutora em Medicina e Ciências da Saúde, docente do Departamento de Patologia, Análises Clínicas e Toxicológicas do Centro de Ciências da Saúde da Universidade Estadual de Londrina. E-mail: reiche@sercomtel.com.br
} 
several genetic polymorphisms, those that occur in the Apo E, Apo B, low-density lipoprotein receptor (LDLR), Apo A-I, Apo C-III, hepatic lipase, cholesteryl ester transfer protein (CETP), lipoprotein lipase (LPL) and proprotein convertase subtilisin/kexin type 9 (PCSK9). have been the object of many studies in the population worldwide. Data on lipid profile and study of polymorphisms of genes encoding structural proteins and enzymes related to lipid metabolism may reveal the prevalence of dyslipidemia in a population, enabling a targeted intervention for the control and prevention of atherosclerotic diseases such as ischemic stroke.

Key words: Stroke. Genetic polymorphism. Dyslipidemia. Low density lipoprotein receptor.

\section{Introdução}

O acidente vascular encefálico (AVE) é definido pelo National Institute of Neurological Disorders and Stroke (NINDS) como uma súbita perda de função cerebral resultante de grave interferência no fluxo de sangue e oxigênio nas artérias cerebrais (PHAN et al., 2002). Cerca de $85 \%$ dos AVE são isquêmicos (AVEi) e decorrentes de uma obstrução arterial encefálica. Dos $15 \%$ restantes, classificados como AVE hemorrágico (AVEh), $10 \%$ são decorrentes de hemorragia intracerebral espontânea e 5\% de hemorragia subaracnóide (ROSAMOND et al., 2008).

O AVE é uma desordem multifatorial decorrente da interação entre componentes genéticos do indivíduo e fatores ambientais. Estudos prévios têm estabelecido que os principais fatores de risco preditores de AVE são hipertensão arterial, tabagismo, diabetes mellitus, dislipidemia, elevado índice de massa corpórea (IMC), distúrbios da coagulação e aumento da idade (ZHANG et al., 2003).

Várias vias fisiológicas estão envolvidas na fisiopatologia do AVEi como o metabolismo lipídico, a resposta inflamatória crônica local e sistêmica, a coagulação, a regulação da pressão sanguínea e a adesão celular e polimorfismos genéticos que ocorrem nestas vias têm sido propostos como fatores genéticos associados ao AVEi (WANG et al., 2009).

Infarto do miocárdio, AVEi e angina pectoris, decorrentes da aterosclerose, são as principais causas de morbidade e mortalidade em adultos nos países desenvolvidos e em desenvolvimento como o Brasil (SANTOS, 2001). São vários os fatores que podem desencadear o processo aterogênico, entre eles dislipidemias, tabagismo, hipertensão arterial, diabetes mellitus, sedentarismo, obesidade e história de doença aterosclerótica prematura (SANTOS, 2001). No entanto, segundo os mesmos autores, a dislipidemia é citada como um dos principais fatores de risco para o desenvolvimento da doença arterial coronariana (DAC).

Os níveis séricos de lipídeos têm etiologia multifatorial e são determinados por um grande número de fatores ambientais e genéticos e a identificação do componente genético na causa da dislipidemia tem sido intensamente investigada nos últimos anos (ANDRADE; HUTZ, 2002). Dentre os fatores genéticos relacionados à DAC estão as variações nos loci genéticos responsáveis pela estrutura e metabolismo de lipoproteínas e do receptor de lipoproteína de baixa densidade $(L D L R)$ que podem contribuir para o desenvolvimento da DAC. Algumas dessas variações genéticas estão associadas com aumento dos níveis séricos de lipídeos e, portanto, podem ser associadas com alto risco para DAC (SALAZAR et al., 1999, 2000b; STEPANOV et al., 1998).

Segundo Salazar et al. (1999), polimorfismos comuns em genes relacionados com o metabolismo lipídico são potencialmente importantes marcadores genéticos para variação no perfil lipídico plasmático, determinando a suscetibilidade ou resistência à DAC na população em geral. O gene $L D L R$ desempenha um papel importante na remoção das partículas de colesterol de lipoproteína 
de baixa densidade (LDL-C) do sangue, regulando a homeostase do colesterol (BROWN; GOLDSTEIN, 1986). Considerando o papel crucial do LDLR na homeostase do colesterol, alterações em seu gene têm contribuído para a variação dos níveis plasmáticos do colesterol na população em geral (SALAZAR et al., 1999, 2000a).

Os dados sobre o perfil lipídico e o estudo dos polimorfismos dos genes que codificam proteínas estruturais e enzimas relacionadas com o metabolismo dos lipídeos podem revelar a prevalência das dislipidemias em uma população, possibilitando uma intervenção direcionada para o controle e prevenção das doenças ateroscleróticas como o AVEi. Considerando a importância da determinação de polimorfismos genéticos como contribuição para o entendimento das causas da dislipidemia, o presente trabalho teve como objetivo revisar os principais polimorfismos genéticos associados ao metabolismo lipídico envolvidos na fisiopatologia do AVEi.

\section{Metodologia}

O presente estudo consiste em uma revisão bibliográfica das informações já publicadas sobre os aspectos relacionados aos fatores genéticos associados com dislipidemias e com o AVEi. Para tanto, foi realizada uma consulta a periódicos nacionais e internacionais e banco de dados virtuais sobre o tema polimorfismos genéticos associados com alterações dos níveis séricos dos lipídeos e AVEi.

Os bancos de dados avaliados foram Literatura Latino-Americana e do Caribe (LILACS), National Library of Medicine (MEDLINE) e Scientific Eletronic Library Online (SCIELO). A partir da pesquisa realizada nas bases de dados, foram selecionados artigos científicos relevantes sobre o tema definido, publicados no período de 1983 a 2014, com a utilização dos seguintes descritores: Acidente vascular encefálico. Polimorfismo genético. Dislipidemia. Receptor de lipoproteína de baixa densidade.

\section{Acidente Vascular Encefálico (AVE)}

O AVE é a maior causa de incapacitação da população acima dos 40 anos de idade. O National Health and Nutrition Examination Survey (NHANES) indica que 6,8 milhões de norteamericanos (2,8\% da população) maiores de 20 anos têm diagnóstico relacionado a AVE. A cada ano, 795.000 pessoas sofrem um episódio de AVE (610.000 casos novos/185.000 casos recorrentes). Isso equivale a um novo AVE a cada 40 segundos nos Estados Unidos da América (EUA). A cada ano, 55.000 mais mulheres que homens têm AVE. Mulheres vivem mais tempo na faixa etária de risco para AVE. Projeções indicam que esse número deve chegar a 10 milhões de vítimas em 2030 nos EUA (GO et al., 2014).

No Brasil, o AVE ocorre com maior frequência na faixa etária superior a 50 anos, sendo responsável por $10 \%$ do total de óbitos, 32,6\% das mortes com causas vasculares e $40 \%$ das aposentadorias precoces no Brasil. O país está entre os dez primeiros com maiores índices de mortalidade por AVE (ABRAMCZUK; VILLELA, 2009), representando a primeira causa de morte por doenças cardiovasculares (FALAVIGNA et al., 2009). Em 2010, segundo dados do Ministério da Saúde, o AVE foi responsável por 52,3 óbitos em 100 mil habitantes, representando $10 \%$ das internações em hospitais públicos (BRASIL, 2013). Os óbitos de pessoas com idades entre 20 e 59 anos corresponderam a $31,1 \%$ do total de óbitos captados pelo Sistema de Informações de Mortalidade (SIM) em 2010, com maior proporção de mortes entre homens e no grupo etário de 50 a 59 anos. As principais causas de morte de adultos com idades entre 20 e 59 anos, no Brasil, foram os homicídios e os acidentes de transporte terrestre (ATTs), seguidos pelas doenças isquêmicas do coração, doenças cerebrovasculares, cirrose e 
outras doenças crônicas do fígado. Apesar disso, a população desconhece a doença, os fatores de risco são mal controlados, poucos centros têm a estrutura e a organização necessárias para atender e tratar, de maneira adequada, os pacientes em fase aguda da doença e poucos locais oferecem reabilitação a estes pacientes (CABRAL, 2009).

O AVE é definido como uma súbita perda de função cerebral resultante de interferência grave no fluxo de sangue e oxigênio nas artérias cerebrais (PHAN et al., 2002). Esta definição distingue o AVE do acidente isquêmico transitório (AIT) que é caracterizado por recuperação completa do quadro em até 24 horas (PATHY; SINCLAIR; MORLEY, 2006). A evolução do AVE dependerá da intensidade lesional do episódio vascular, da importância funcional da área cerebral envolvida e da qualidade do atendimento de emergência prestado (MCPHEE; PAPADAKIS; TIERNEY, 2007).

AVE é uma desordem complexa, multifatorial e poligênica decorrente da interação entre componentes genéticos do indivíduo e fatores ambientais, e estudos têm estabelecido que hipertensão arterial, tabagismo, diabetes mellitus, dislipidemia, elevado índice de massa corpórea (IMC), distúrbios da coagulação e aumento da idade como alguns dos fatores de risco preditores para o desenvolvimento de AVE (ZHANG et al., 2003). O AVEi apresenta diferentes etiologias, tais como, doença aterosclerótica de grandes artérias, doença de pequenos vasos, cardioembolia, vasculites, trombofilias, anemia falciforme, dissecção arterial e doenças genéticas. No entanto, em até $30 \%$ dos casos, a etiologia não pode ser definida (ROSAMOND et al., 2008).

Dentre os fatores de risco não modificáveis que contribuem para o AVEi, o mais importante é a idade, havendo clara relação do envelhecimento com o risco de se ter AVEi, que aumenta por volta dos 60 anos e duplica a cada década. Outros fatores não modificáveis são hereditariedade, sexo e etnia; o sexo masculino e a etnia negra mostram maior incidência de AVEi (PIRES et al., 2004). Entre os fatores de risco modificáveis, a hipertensão arterial é o mais relevante, ocasionando aumento superior a sete vezes na incidência de AVEi. As doenças cardíacas, sobretudo as arritmias potencialmente emboligênicas e, em meio a elas, a fibrilação atrial e diabetes são também fatores de risco relevantes. Tabagismo é outro fator determinado que eleva o risco relativo em $50 \%$. Sedentarismo, estresse, obesidade, dislipidemia e uso de contraceptivos orais são também fatores de risco comprovados. Vale ainda evidenciar o risco potencial de AVEi em procedimentos hemodinâmicos e cirurgias cardiológicas (PIRES et al., 2004).

Várias vias fisiológicas como do metabolismo lipídico, da resposta inflamatória crônica local e sistêmica, da coagulação, da regulação da pressão sanguínea e da adesão celular têm sido implicadas na fisiopatologia do AVEi, e polimorfismos genéticos que ocorrem nestas vias têm sido propostos como fatores genéticos associados ao AVEi (WANG et al., 2009). Infecções bacterianas e virais são fatores de risco independentes para o AVEi e aumento dos níveis de marcadores inflamatórios, como a proteína $\mathrm{C}$ reativa (PCR), contagem de leucócitos periféricos e dosagem de fibrinogênio, está associado com aumento do risco de AVEi (GRAU et al., 1996).

\section{AVE e Principais Fatores Genéticos Envolvidos}

Dados epidemiológicos fornecem evidência substancial que o componente genético está associado ao desenvolvimento do AVEi. Grande progresso tem sido feito no estudo da identificação de um único gene associado com AVEi. A identificação de mutações no gene NOTCH3 (Neurogenic locus notch homolog protein 3) em pacientes com arteriopatia autossômica dominante cerebral com infartos subcorticais e leucoencefalopatia (CADASIL) levou a novas descobertas sobre AVEi lacunar e doença de 
pequenos vasos. Estudos sobre doença falciforme têm chamado a atenção para a importância de genes modificadores e das interações entre genes na determinação do risco de AVEi e doença de pequenos vasos. Destacaram ainda um potencial papel da genética na previsão de risco de AVE (DICHGANS, 2007).

Estudos de associação genética com AVEi têm revelado uma ampla variedade de genes candidatos que codificam moléculas envolvidas no sistema de coagulação sanguínea, na resposta inflamatória, no metabolismo de lipídeos, carboidratos e homocisteína, na regulação do ciclo celular e no sistema renina-angiotensina. A contribuição genética ao AVEi parece ser poligênica e, provavelmente, há vários alelos que exercem pequenos efeitos (risco relativo $<1,5$ ), mas devido à ampla distribuição na população em geral, o impacto na fiosiopatologia do AVEi é grande (DICHGANS, 2007). Vários polimorfimos genéticos são considerados de risco para o AVEi, conforme ilustra a figura I.

Estudos de todo o genoma na população da Islândia levaram à identificação dos genes fosfodiesterase 4D (PDE4D), cAMC especifico e proteina ativadora da aracdonate 5-lipoxigenase (ALOX5AP - Arachidonate 5-LipoxygenaseActivating Protein). Haplótipos específicos nestes genes têm sido associados com o aumento do risco para AVEi na população da Islândia, mas o seu papel em outras populações não é claro (DICHGANS, 2007).

Estudos com gêmeos, famílias e modelos animais provaram uma forte evidência da contribuição genética para a ocorrência de AVEi. No entanto, a extensão da predisposição genética é desconhecida (TONK; HAAN, 2007). Segundo Tonk e Haan (2007), atribuir fatores genéticos que desempenham um papel em comum no AVE é muito difícil, pois a causa do AVE é multifatorial, ou seja, uma combinação de fatores de riscos ambientais e genéticos e, a parte genética é muito complexa, pois, envolve múltiplos genes desempenham um papel (poligênica). Muitos fatores de riscos comuns para o AVEi, como diabetes mellitus e hipertensão arterial são, em parte, herdados, e muitos loci genéticos podem contribuir para um fenótipo de maior ou menor risco para o desenvolvimento de AVE.

Estudos recentes encontraram um aumento das formas monogênicas de AVEi e AVEh, como o CADASIL e angiopatias amilóides, respectivamente. Esses distúrbios podem servir como modelos para estudar os fatores ambientais ou genéticos que contribuem também para as formas comuns de AVE. Estudos em modelo animal de AVE também podem fornecer informações valiosas sobre os fatores genéticos envolvidos na predisposição a esta doença (TONK; HAAN, 2007).

Yamada et al. (2006) identificaram polimorfismos genéticos relacionados ao risco de AVEi e AVEh em 3151 indivíduos japoneses, sendo 1141 pacientes que sofreram AVE (636 com AVEi, 282 com AVEh intracerebral e 223 com AVEh subaracnóide) e 2010 indivíduos controles. Os resultados demonstraram que o polimorfismo no gene interleucina 6 (IL6) pode ser útil na avaliação do risco genético para AVEi e AVEh intracerebral. Determinados genótipos para os genes transportador de membrana não acoplado à proteina 3 (UCP3), fator de necrose tumoral (TNF) e doença renal policística 1-like (PKD1like) podem ser igualmente úteis na avaliação do risco de AVEh subaracnóide (YAMADA et al., 2006).

Em estudo do polimorfismo genético da apoproteína $E$ (Apo E), o genótipo E3/E3 apresentou papel protetor contra a ocorrência do AVEi, especialmente em pacientes do sexo feminino. No entanto, fatores de risco como idade, IMC, hipertensão arterial, dislipidemia, diabetes mellitus e tabagismo apresentaram um forte impacto sobre a ocorrência do AVEi e superaram o papel protetor 
do genótipo $\varepsilon 3 / \varepsilon 3$ (CHATZISTEFANIDIS et al., 2014).

Os dados sobre o perfil lipídico e o estudo dos polimorfismos dos genes que codificam proteínas estruturais e enzimas relacionadas com o metabolismo dos lipídeos podem revelar a prevalência das dislipidemias em uma população, possibilitando uma intervenção direcionada para o controle e prevenção das doenças ateroscleróticas como o AVEi.

Figura 1 - - Diferentes polimorfismos genéticos envolvidos na etiopatogenia do acidente vascular encefálico (AVE).

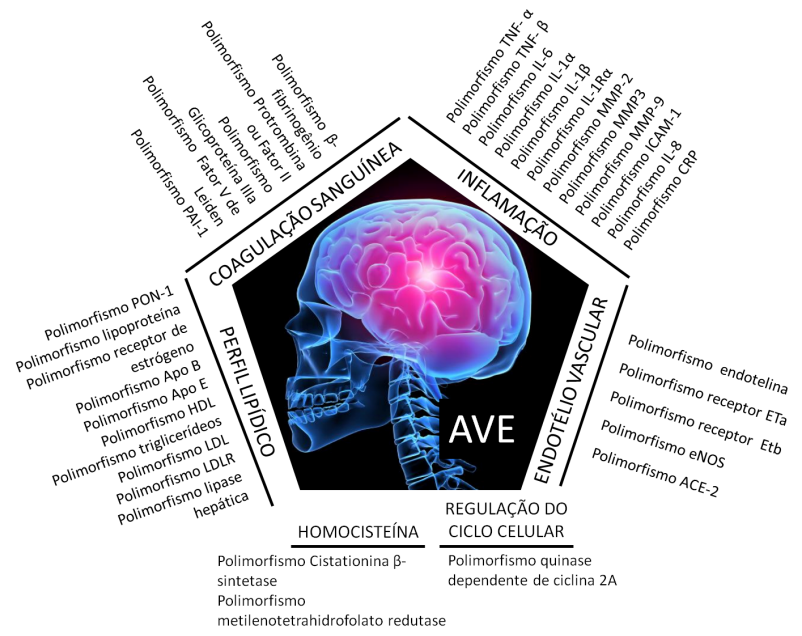

PAI-1: inibidor do ativador do plasminogênio 1; PON1: paraoxonase 1; Apo: apoproteína; HDL: lipoproteína de alta densidade; LDL: lipoproteína de baixa densidade; LDLR: receptor de lipoproteína de baixa densidade; TNF: fator de necrose tumoral; IL: interleucina; MMP: metaloproteinases de matriz; ICAM: molécula de adesão intercelular; CPR: proteína $\mathrm{C}$ reativa; ETa: receptor de endotelina tipo A; ETb: receptor de endotelina tipo B; eNOS: óxido nítrico sintetase endotelial; ACE-2: enzima de conversão da angiotensina.

Fonte: Autores.

\section{Polimorfismos Genéticos Associados à Dislipidemia}

Vias fisiológicas do metabolismo lipídico têm sido implicadas na fisiopatologia do AVEi e polimorfismos genéticos que ocorrem nestas vias têm sido propostos como fatores genéticos associados ao AVEi (WANG et al., 2009). Os níveis séricos de lipídeos têm etiologia multifatorial, determinada por um grande número de fatores ambientais e genéticos (ANDRADE; HUTZ, 2002). Fatores genéticos e da dieta influenciam a concentração sérica de colesterol total (CT), mas mecanismos detalhados de sua interação não são bem conhecidos. $\mathrm{O}$ aumento da ingestão de colesterol na dieta aumenta a concentração sérica de colesterol em alguns indivíduos, mas não em outros. No entanto, este mecanismo ainda não está compreendido claramente. Variações genéticas em Apo, enzimas e receptores que atuam, principalmente, no metabolismo do LDL-C estão envolvidas, pelo menos em parte, na regulação da concentração sérica de CT e LDL-C (SALAZAR et al., 2000b). Segundo Andrade e Hutz (2002), a identificação do componente genético na causa da dislipidemia tem sido intensamente investigada nos últimos anos. Estudos demonstram que o efeito desses polimorfismos depende em parte da interação com fatores ambientais, tais como tabagismo, sobrepeso ou sedentarismo.

Polimorfismos em um grande número de genes estão envolvidos na síntese de proteínas estruturais e enzimas relacionadas com o metabolismo de lipídeos poderiam responder por variações do perfil lipídico de cada indivíduo (ALMEIDA et al., 2013; ANDRADE; HUTZ, 2002). Tais variações genéticas, quando encontradas com frequência na população estudada (mais de 1\% de frequência do alelo mais raro), são chamadas de polimorfismos genéticos. Desta maneira, qualquer gene que participe da produção de uma proteína envolvida no metabolismo dos lipídeos poderia ser um candidato para investigação de determinantes genéticos dos níveis lipídicos (ANDRADE; HUTZ, 2002).

Polimorfismos nos genes da Apo E, Apo B, LDLR, Apo A-I, Apo C-III, lipase hepática, proteina transferidora de ésteres de colesterol 
(CETP), lipase lipoprotéica (LPL) e proteina convertase subtilisina/kexina tipo 9 (PCSK9) foram estudados e relacionados com variações no perfil lipídico de diferentes populações. A tabela I sumariza alguns dos polimorfismos genéticos associados aos níveis séricos de lipídeos.

A Apo E compõe a estrutura da lipoproteína de alta densidade (HDL-C), lipoproteína de densidade muito baixa (VLDL) e quilomícrons, além dos produtos de degradação lipolítica, como remanescentes de quilomícrons e lipoproteína de densidade intermediária (IDL). Essa proteína plasmática atua como fator de ligação com os receptores celulares. Além disso, tem um reconhecido papel no transporte do colesterol e de outros lipídeos dos tecidos periféricos para o fígado, a fim de que possam ser metabolizados (BRANDÃO et al., 2004).

A Apo $E$ desempenha importante papel no catabolismo destas lipoproteínas ricas em triglicerídeos (TG) e no transporte do colesterol em vários tecidos. O gene Apo E localiza-se no braço longo do cromossomo 19 e codifica uma proteína de 299 aminoácidos (FORTI et al., 2003). Segundo Andrade e Hutz (2002), o gene Apo $E$ exerce forte influência sobre os níveis séricos de LDL-C.

O gene Apo $E$ possui um polimorfismo comum, o HhaI (T112C, rs429358), localizado no exon 4 que gera três alelos: $\varepsilon 2$, $\varepsilon 3$ e $\varepsilon 4$ (FORTI et al., 2003). A frequência destes alelos varia de acordo com cada população (HALLMAN et al., 1991). Estudos demonstram que o alelo $\varepsilon 3$ é o mais comum em várias populações (EICHNER et al., 2002). Segundo Schwanke et al. (2002), o polimorfismo do Apo E modifica a proteína tanto na sua estrutura quanto na sua função. De acordo com Siest et al. (1995), as isoformas da Apo $E$ interagem de maneira diferente com os receptores de lipoproteínas, alterando seu metabolismo e, consequentemente, os níveis de lipídeos circulantes no plasma.
Tabela 1 - Polimorfismos genéticos associados com níveis séricos de lipídeos em diferentes populações.

\begin{tabular}{|c|c|c|c|c|c|}
\hline Gene (Polimorfismo) & Cromossomo & Região & $\begin{array}{c}\text { Alelo } \\
\text { relacionado }\end{array}$ & Frequência ${ }^{2}$ & $\begin{array}{l}\text { Porção lipídica } \\
\text { modificada }\end{array}$ \\
\hline Apo E (HhaI, rs429358) & 19 & exon 4 & 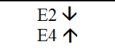 & $\begin{array}{l}\sim 8 \% \\
\sim 15 \%\end{array}$ & CT e LDL-C \\
\hline LDLR $(P v u \amalg I, \mathrm{C}>\mathrm{T})$ & 19 & intron 15 & $P-\uparrow$ & $\sim 20 \%$ & \\
\hline LDLR (AvaII, rs5925) & 19 & exon 13 & $A+\uparrow$ & $\sim 56 \%$ & \\
\hline LDLR (HincII, rs688) & 19 & exon 12 & $\mathrm{H} \uparrow$ & $\sim 56 \%$ & \\
\hline Apo B (XbaI, rs693) & 2 & exon 26 & $x+\uparrow$ & $\sim 40 \%$ & \\
\hline Apo B (ins/del, rsl 7240441$)^{3}$ & 2 & exon 1 & $\operatorname{del} \uparrow$ & $\sim 35 \%$ & \\
\hline LPL (HindIII, rs320) & 8 & intron 8 & $\mathrm{H}+\uparrow$ & $\sim 65 \%$ & TG \\
\hline$L P L(P v u I I, \mathrm{rs} 285)$ & 8 & intron 6 & $\mathrm{P}+\uparrow$ & $\sim 50 \%$ & \\
\hline LPL (D9N, rs1801177) & 8 & exon 2 & N9 个 & $-3 \%$ & \\
\hline LPL (S291N, rs268) & 8 & exon 6 & 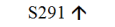 & $-4 \%$ & \\
\hline$L P L(\mathrm{~S} 447 \mathrm{X}, \mathrm{rs} 328)^{4}$ & 8 & exon 9 & $\mathrm{X} 447 \downarrow$ & $\sim 20 \%$ & \\
\hline Apo C-III (SstI, rs10892152) & 11 & 3'UTR & $\mathrm{S}+\uparrow$ & $12 \%$ & \\
\hline Apo A-I (MspI, rs5069) & 11 & intron 1 & $\begin{array}{c}-75 \mathrm{~A} \uparrow \\
\mathrm{M}-\uparrow\end{array}$ & $\begin{array}{l}\sim 20 \% \\
\sim 4 \%\end{array}$ & HDL-C \\
\hline CETP (Taq IB, rs 708272) & 16 & intron 1 & $\begin{array}{l}T+\downarrow \\
T-\uparrow\end{array}$ & $-40 \%$ & \\
\hline LIPC (NlaIII, rs 1800588$)$ & 15 & promotora & $\begin{array}{l}-514 \mathrm{C} \downarrow \\
-514 \mathrm{~T} \uparrow\end{array}$ & $\sim 80 \%$ & \\
\hline
\end{tabular}

$1 \uparrow$ relação com aumento dos níveis; $\downarrow$ relação com diminuição dos níveis; + denota presença do sítio de restrição, enquanto - denota ausência do sítio; 3'UTR: região 3' não transcrita.

2 Médias aproximadas das frequências alélicas dentre várias populações.

${ }^{3}$ ins denota alelo de inserção e del alelo de deleção.

${ }^{4} \mathrm{X}$ denota stop códon

Fonte: Adaptado de Andrade e Hutz (2002).

De acordo com Davignon, Gregg e Sing (1988), em países industrializados, indivíduos que carregam o alelo $\varepsilon 4$ possuem altos níveis séricos de CT e LDL-C, enquanto indivíduos com o alelo ع3 possuem níveis intermediários e aqueles que carregam o alelo $\varepsilon 2$ possuem os menores níveis. Segundo Hallman et al. (1991), a associação entre o alelo $\varepsilon 4$ e níveis elevados de CT e LDL e entre o alelo $\varepsilon 2$ e baixos níveis desses lipídeos tem sido documentada por vários estudos, independente do grupo étnico avaliado. Mendes-Lana et al. (2007) demonstraram em indivíduos de Ouro Preto, São Paulo, que o alelo $\varepsilon 2$ pode conferir um efeito protetor; entretanto, a presença do alelo $\varepsilon 4$ implicou em aumento do risco para dislipidemia.

A Apo $B$ é a principal proteína constituinte do LDL-C e VLDL, sendo sintetizada no fígado e intestino. A Apo B é essencial para a montagem, secreção e metabolismo das partículas lipoprotéicas e também para remoção do LDL-C da circulação através do LDLR (BROWN; GOLDSTEIN, 1986). 
A regulação da concentração plasmática do $\mathrm{CT}$ e LDL-C são mediadas pela ligação da $A p o B$, presente na partícula de LDL-C, com o $L D L R$ na superfície celular, ou seja, a Apo $B$ capta as partículas ricas em colesterol do plasma pela endocitose mediada pelos $L D L R$ presentes na superfície celular (BROWN; GOLDSTEIN, 1986).

Alterações genéticas e estruturais na $A p o B$ têm sido associadas com um defeito de sua ligação com o $L D L R$ levando à hipercolesterolemia, um importante fator de risco para aterosclerose e DAC prematuras (LEWIS, 1983).

O gene Apo $B$ está situado no cromossomo 2 e nele já foram descritas algumas mutações e vários polimorfismos (FORTI et al., 2003). Em várias populações, polimorfismos neste gene estão associados a variações nas concentrações plasmáticas de lipídeos e DAC ou infarto do miocárdio (GARDEMANN et al., 1998) e variação dos níveis séricos de $\mathrm{CT}$ e com a aterosclerose coronariana (GUZMÁN et al., 2001). Os polimorfismos inserção/deleção Ins/Del (rs17240441) no exon 1, MspI (rs676210) e XbaI (rs693) no exon 26 e 3'HVR têm sido associados com variações nos níveis de lipídeos, DAC e infarto do miocárdio (PEACOCK et al., 1992). Sakuma, Hirata e Hirata (2004) demonstraram associação do polimorfismo EcoRI (rs1042031) no exon 29 com elevados níveis de LDL-C. Variações nos níveis de CT e TG, obesidade e DAC têm sido também demonstradas (STEPANOV et al., 1998).

A proteína transferidora de ésteres de colesterol (CETP)possuiumpapelfundamentalnometabolismo do HDL-C, promove a troca de lipídeos entre as lipoproteínas, transferindo ésteres de colesterol do HDL-C para outras lipoproteínas para subsequente absorção do colesterol pelos hepatócitos. A transferência de ésteres de colesterol para o LDL-C e VLDL ocorre em troca de TG (BARTER, 2002). Este fluxo do colesterol para o fígado é conhecido como transporte reverso do colesterol. Por aumentar a quantidade de ésteres de colesterol no LDL-C e
VLDL, a CETP aumenta a aterogenicidade dessas lipoproteínas. Concentrações plasmáticas elevadas de CETP estão associadas com a redução do HDL-C sendo um forte e independente fator de risco para a aterosclerose (KUIVENHOVEN et al., 1998). Vários pesquisadores têm mostrado que o papel da CETP depende do contexto metabólico e pode ser afetado por outras enzimas e proteínas do plasma que são cruciais no metabolismo das lipoproteínas (FIELDING; HAVEL, 1996). Polimorfismos no gene CETP (TaqIB, rs708272) são responsáveis por variações na atividade da CETP e influenciam as concentrações plasmáticas de HDL-C (KUIVENHOVEN et al., 1998).

A lipase lipoproteica $(L P L)$ está estrategicamente ancorada ao endotélio vascular e desempenha um papel central no metabolismo lipídico. A $L P L$ catalisa a hidrólise dos TG, constituindo o passo limitante na remoção da circulação de lipoproteínas ricas em TG, tais como quilomícrons, VLDL e LDL-C (ECKEL, 1989). Tem sido demonstrado que a $L P L$ atua como um ligante para o $L D L R$ influenciando a secreção hepática e captação de VLDL e LDL-C (MULDER et al., 1993).

Mutações no gene $L P L$ são conhecidas por causarem dislipidemia e estão associadas à aterosclerose prematura (BENLIAN et al., 1996). Variantes polimórficas do gene $L P L$ foram identificadas e avaliadas por seus efeitos sobre os lipídeos plasmáticos e risco de aterosclerose (PEACOCK et al., 1994). Os polimorfismos HindIII (rs320) e PvuII (rs285) localizados nos introns 8 e 6 , respectivamente, são comuns e podem ser associados com alterações plasmáticas nos lipídeos e, consequentemente, associados com a DAC (PEACOCK et al., 1992).

O gene Apo $C$-III = codifica a Apo $C$ - $I I I=$, principal apoproteína das lipoproteínas ricas em TG (quilomicrons e VLDL) (PORKKA et al., 1994). Os polimorfismos nesse gene parecem influenciar os níveis séricos de TG (DALLONGEVILLE et al., 2000). Vários estudos têm encontrado uma 
associação entre a presença do sítio polimórfico SstI (rs10892152) na região 3' não transcrita (3’UTR) e aumento na concentração de Apo C-III $=$ e $\mathrm{TG}$ e com um risco aumentado para DAC (DALLONGEVILLE et al., 2000; FIEGENBAUM; ANDRADE; HUTZ, 2007).

A Apo A possui quatro isoformas (A-I, A-II, A-IV e A-V) que fazem parte da constituição do HDL-C, lipoproteína com função antioxidante e responsável pelo transporte reverso do colesterol. A Apo A-I é cofator da lecitina colesterol acil transferase (LCAT), enzima do metabolismo reverso do colesterol, e polimorfismos no gene Apo $A-I$ estão relacionados com baixas concentrações de HDL-C (JENNER et al., 2005). O polimorfismo MspI (rs5069) foi associado com elevados níveis de Apo A-I e HDL-C (PEACOCK et al., 1994).

Variações no gene LIPC que codifica a lipase hepática têm sido associadas aos níveis de HDL-C. A lipase hepática está envolvida no metabolismo de várias lipoproteínas e tem um papel fundamental no transporte reverso do colesterol (COUTURE et al., 2000). Um polimorfismo comum decorrente da substituição do nucleotídeo citosina por timina na posição -514 da região promotora do gene LIPC (NlaIII, rs1800588) tem sido associado com variações plasmáticas nos níveis de HDL-C e atividade da enzima (COUTURE et al., 2000). Em um estudo com 1314 homens e 1353 mulheres, Couture et al. (2000) demonstraram que indivíduos que carregavam o alelo $-514 \mathrm{~T}$ possuíam elevadas concentrações de Apo A-I e HDL-C devido ao aumento da subfração HDL2-C.

Outro gene relacionado à dislipidemia é o que codifica a protease pró-proteina convertase subtilisina/kexina tipo 9 (PCSK9). A PCSK9 é o nono membro da família das proteínas convertase e é relacionada com a proteinase K. Esta enzima está envolvida no metabolismo do colesterol induzindo a degradação do $L D L R$ e o aumento da sua atividade leva ao decréscimo do número de $L D L R$ disponíveis para a captação do colesterol plasmático com consequente aumento das concentrações séricas de LDL-C (MAXWELL; FISHER; BRESLOW, 2005). Mutações no gene PCSK9, localizado no cromossomo 1p32, levam a enfermidades com quadro clínico idêntico a Hipercolesterolemia Familar (HF) (ABIFADEL et al., 2003). O polimorfismo R46L (rs11591147) no PCSK9 diminui as concentrações de LDL-C e CT, podendo causar um efeito protetor do infarto do miocárdio (SCARTEZINI et al., 2007).

Em um estudo de meta-análise de 46 estudos genômicos de associação, outros 95 loci foram identificados como fatores genéticos que contribuem com as variações normais dos lipídeos e com os fenótipos extremos de dislipidemias na população em geral (TESLOVICH et al., 2010). Estudos de epidemiologia molecular realizados em diferentes populações têm mostrado a elevada frequência de variantes nos genes $L D L R$, Apo B e PCSK9 em pacientes com HF e seus probandos (MARDUEL et al., 2010).

Marduel et al. (2010) avaliaram 1358 probandos de diferentes regiões da França e identificaram 1111 eventos moleculares: $1012(91,1 \%)$ mutações no $L D L R$ em 1003 (73,9\%) probandos, 9 com duas variantes do $L D L R$ cada; 89 (8,0\%) mutações no Apo B em $89(6,6 \%)$ probandos incluindo 2 probandos também heterozigotos para mutação no LDLR; 10 (0,9\%) mutações no PCSK9 em 10 (0,7\%) probandos. Para os demais $258(19,0 \%)$ probandos, os autores não identificaram mutações nos três principais genes associados à hipercolesterolemia autossômica dominante (ADH), o que reforça a existência de outras mutações associadas à ADH em genes ainda não identificados. Das mutações no $L D L R, 46,0 \%$ foram missense, $14,6 \%$ causaram alterações no quadro de leitura (frameshift), 13,6\% alteraram o splicing, $11,3 \%$ foram mutações de códon de parada (nonsense), 9,7\% foram rearranjos maiores, 3,8\% foram inserções/deleções (ins/del) e $1,0 \%$ das mutações ocorreram na região 3'URT. Marduel et al. (2010) ainda descrevem que todas as mutações não sinônimas e de mudança do quadro de 
leitura foram consideradas causadoras da $\mathrm{FH}$ uma vez que têm com consequência a síntese de uma proteína truncada. Das 20 novas variações intrônicas no $L D L R, 14$ (70\%) foram preditas serem deletérias e seis (30\%) foram preditas serem benignas, segundo estudos in vitro funcionais e análises in sílica.

\section{Polimorfismo no gene LDLR}

O LDLR desempenha um papel importante na remoção das partículas de LDL-C do sangue, regulando a homeostase do colesterol. Muitas mutações no gene $L D L R$ têm sido associadas com a disfunção do $L D L R$, que reduz significativamente o catabolismo de LDL-C, levando à desordem metabólica de HF. Pacientes com mutações no gene $L D L R$ possuem níveis plasmáticos de colesterol elevados duas ou mais vezes que a concentração normal, o que aumenta o risco de desenvolvimento de aterosclerose e DAC (BROWN; GOLDSTEIN, 1986).

O LDLR modula os níveis plasmáticos de LDL-C regulando a absorção das partículas de LDL-C pelo fígado e entrega de colesterol para as glândulas suprarrenais e gônadas para a síntese de hormônios esteroides e para o fígado para a síntese de ácidos biliares (BROWN; GOLDSTEIN, 1986). Considerando o papel crucial do $L D L R$ na homeostase do colesterol, alterações genéticas no gene $L D L R$ têm contribuído para a variação dos níveis plasmáticos do colesterol na população em geral (SALAZAR et al., 1999).

O gene $L D L R$ localiza-se no cromossomo 19 , compreende 18 exons e 17 introns e codifica uma proteína de 839 aminoácidos (FORTI et al., 2003). A natureza polimórfica deste gene já foi demonstrada pelo método de polimorfismo do comprimento dos fragmentos de restrição (RFLP) (BERTOLINI et al., 1992).

Já foram descritas mais de 1288 variantes diferentes para o LDLR em pacientes com HF: 55\% destas variantes correspondem a substituições em regiões de exons, $22 \%$ são pequenos rearranjos em exons com menos de 100 pares de bases (pb), 11\% são grandes rearranjos com mais de 100 pb, 2\% ocorrem em regiões promotoras, $10 \%$ em introns e $1 \%$ são variações na região 3' UTR (LEIGH et al., 2008; USIFO et al., 2012).

Vários SNPs do $L D L R$ foram avaliados com o uso de enzimas de restrição como o RsaI (extremidade 5'), StuI (exon 2), MaeIII (exon 4), TaqI (intron 4), SphI (intron 6), StuI (exon 8), HhaI (exon 11), HincII (rs688, exon 12), AvalI (rs5925, exon 13), MspI (exon 15), PvuII (intron 15), MspI e NcoI (exon 18) e PstI (extremidade 3') (VILLEGER et al., 2002). Estudos relataram a associação entre o polimorfismo $P v u$ II $(\mathrm{C}>\mathrm{T})$ localizado no intron 15 do $L D L R$ com diferentes níveis de LDL-C, onde indivíduos com o genótipo homozigoto $\mathrm{P} 2 \mathrm{P} 2$ (presença do sítio de restrição da enzima), apresentaram uma redução de 10-20\% dos níveis de LDL-C comparados com os indivíduos com outros genótipos (PEDERSEN; BERG, 1988, 1989; SCHUSTER et al., 1990).

Salazar et al. (1999) realizaram um estudo sobre os efeitos do polimorfismo AvalI e HincII do $L D L R$ nos níveis séricos de lipídeos em indivíduos brasileiros com alto risco para DAC (170 indivíduos considerados com alto risco para DAC e 130 indivíduos clinicamente normais). A frequência dos alelos $\mathrm{A}+$ (AvaII) e $\mathrm{H}+$ (HincII) foi maior em indivíduos com alto risco para DAC quando comparada com as obtidas nos indivíduos do grupo controle ( $56 \%$ vs $45 \%$, para os dois alelos, $\mathrm{p}=0,01505)$; além disso, essa frequência foi maior que a observada em outros estudos com indivíduos caucasianos hipercolesterolêmicos de Londres, Itália, Espanha, Suíça e Alemanha (HUMPHRIES et al., 1993).

A frequência dos genótipos $\mathrm{A}+\mathrm{A}+$ e $\mathrm{H}+\mathrm{H}+$ encontrada por Salazar et al. (1999) foi maior em indivíduos com alto risco para DAC quando comparada ao grupo de indivíduos clinicamente normais (32\% vs $16 \%$ e $32 \%$ vs $18 \%$ respectivamente). Além disso, indivíduos com 
genótipo homozigoto $\mathrm{A}+\mathrm{A}+$ apresentaram altos níveis de CT e LDL-C em indivíduos com alto risco para DAC quando comparados aos indivíduos com outros genótipos. Resultados similares foram encontrados para o polimorfismo HincII, em que o genótipo $\mathrm{H}+\mathrm{H}+$ foi associado com maiores níveis séricos de CT e LDL-C em indivíduos com alto risco para DAC. Os efeitos dos polimorfismos AvaII e HincII não foram observados em indivíduos do grupo controle. No entanto, em estudos anteriores não foi detectado uma significativa associação entre os diferentes genótipos destes polimorfismos no gene $L D L R$ e níveis séricos de lipídeos (TAYLOR et al., 1988).

A forte associação entre os genótipos $\mathrm{A}+\mathrm{A}+$ (AvaII) e $\mathrm{H}+\mathrm{H}+$ (HincII), encontrados por Salazar et al. (1999), com o aumento dos níveis séricos circulantes do CT e LDL-C nos indivíduos com alto risco para DAC fornece uma importante evidência que estes polimorfismos no $L D L R$ afetam os níveis de colesterol nesses indivíduos. Além disso, vários estudos têm indicado que este polimorfismo comum no gene $L D L R$ é associado a diferenças nos níveis plasmáticos de LDL-C em indivíduos normo e hipercolesterolêmicos (BERTOLINI et al., 1992; GUDNASON et al., 1998).

O polimorfismo $P v u I I$ localizado no intron 15 do gene $L D L R$ tem sido considerado um marcador genético ligado à variação do $L D L R$ que altera estruturalmente a atividade do receptor ou altera sua função regulatória (GUDNASON et al., 1998). Estudos têm demonstrado que o polimorfismo $P v u I I$ foi associado com diferenças na concentração do LDL-Cemindivíduosnormoehipercolesterolêmicos de diferentes países (BERTOLINE et al., 1992). No entanto, Taylor et al. (1988) não demonstraram associação significativa entre os genótipos do $L D L R$ e concentrações séricas de lipídeos.

Salazar et al. (2000b) demonstraram a influência do polimorfismo $P v u I I$ do $L D L R$ nas concentrações séricas de lipídeos em indivíduos com baixo e alto risco para DAC. Foram analisados 128 indivíduos caucasianos brasileiros com perfil lipídico sugestivo para DAC e 100 indivíduos normolipêmicos. A frequência do alelo P- (ausência de sítio de restrição) em indivíduos com alto risco para DAC foi de $75 \%$, similar à observada em indivíduos hipercolesterolêmicos da Itália, Suíça, Alemanha, Israel, Espanha, Londres, Holanda, Dinamarca e América do Norte (BERTOLINI et al., 1992).

O genótipo P-P- do polimorfismo PvuII foi mais frequente em indivíduos com alto risco para DAC quando comparado aos controles (57\% vs 38\%, p<0,05). Além disso, os autores demonstraram uma forte associação entre elevada concentração plasmática de CT, TG, LDL-C, VLDL e baixa concentração plasmática de HDL-C em indivíduos com alto risco para DAC. Similarmente, demonstraram que indivíduos do grupo controle (baixo risco para DAC) com o genótipo P-Papresentavam aumento da concentração de CT e LDL-C quando comparados aos indivíduos com outros genótipos (P-P+e P+P+) (SALAZAR et al., 2000b).

Em estudos com mulheres caucasianas brasileiras com DAC, Salazar et al. (2000c) mostraram que a frequência dos genótipos homozigotos $\mathrm{A}+\mathrm{A}+\mathrm{e}$ P-P- para os polimorfismos AvaII e PvuII no gene $L D L R$ foi significantemente maior em mulheres com DAC do que no grupo controle (44\% vs $16 \%$ e $64 \%$ vs $39 \%, p<0,05$, respectivamente). Além disto, a frequência dos alelos A+ e P- encontrados em mulheres com DAC também foi maior do que no grupo controle ( $62 \%$ vs $44 \%$, $p=0,005$ e $78 \%$ vs $65 \%, p<0,05$, respectivamente). No entanto, para o polimorfismo HincII não foi demonstrada diferença significativa entre pacientes e controles.

Salazar et al. (2000a) avaliaram os polimorfismos AvaII (exon 13), HincII (exon 12) e PvuII (intron 15 do gene $L D L R$ ) em 50 indivíduos brasileiros diagnosticados com $\mathrm{HF}$ e 130 indivíduos normolipêmicos. Os indivíduos com HF mostraram maior frequência dos genótipos homozigotos $\mathrm{A}+\mathrm{A}+$ (AvaII), $\mathrm{H}+\mathrm{H}+$ (HincII) e P-P- (PvuII) 
quando comparados ao grupo controle $(\mathrm{p}<0,05)$. Indivíduos com HF apresentaram maior frequência dos alelos $\mathrm{A}+(58 \%), \mathrm{H}+(61 \%)$ e $\mathrm{P}-(78 \%)$ do que os indivíduos normolipêmicos $(45 \%, 45 \%$ e $64 \%$, respectivamente). A forte associação observada entre esses alelos e HF sugere que os polimorfismos AvaII, HincII e PvuII podem ser utilizados para monitorar a hereditariedade para HF em famílias brasileiras.

Com relação à resposta terapêutica aos medicamentos inibidores da enzima 3-hidroxi-3metilglutaril-coenzymaA (HMG-CoA) redutase utilizados no tratamento da hipercolesterolemia, Salazar et al. (2000d) demonstraram a associação de polimorfismos no $L D L R$ com a resposta terapêutica à fluvastatina em 55 pacientes brasileiros com hipercolesterolemia primária. Os resultados indicaram que os polimorfismos AvaII e PvuII influenciam a resposta terapêutica à fluvastatina. Indivíduos com os genótipos homozigotos $\mathrm{A}+\mathrm{A}+$ (AvaII) e P-P- (PvuII) apresentaram menor redução dos níveis de CT, LDL-C e Apo $B$ após 16 semanas de tratamento com fluvastatina, quando comparados aos outros genótipos. Não foram encontradas diferenças significativas com relação ao polimorfismo HincII.

Polimorfismos genéticos no $L D L R$ têm sido associados com AVEi em diferentes populações (WANG et al., 2009). Em um estudo com pacientes de Taiwan que sofreram AVE foi constatado que os polimorfismos genéticos rs2738446 e rs2738450 no $L D L R$ podem modificar a apresentação do AVEi (LEE et al., 2012). O LDL-C transita através da barreira hematoencefálica mediado pelo $L D L R$. No cérebro, o $L D L R$ é expresso, principalmente, nas células endoteliais dos capilares e, portanto, é considerado como um gene de susceptibilidade importante para modificar a apresentação do curso do AVEi.

Lee et al. (2012) analisaram o papel potencial de duas variantes do LDLR, (Hinc I, rs688 e AvaII, rs5925, no AVEi. Este estudo de associação genética foi realizado em uma população de Taiwan com 815 pacientes que sofreram AVEi (431 com AVE aterotrombótico e 384 com infarto lacunar) e 430 controles normais. Não houve diferença significativa na frequência genética dos polimorfismos rs688 e rs5925 entre o grupo controle e AVEi em geral, grupo aterotrombótico ou grupo de infarte lacunar. No entanto, quando a análise de associação de haplótipos entre as relacionadas com rs688 e rs5925 e AVEi, o alelo mais comum, haplótipo $\mathrm{CT}$, foi usado como o alelo de referência, e o haplótipo TC associado a um risco acrescido de 65\% de AVEi global, 72\% aumento do risco de AVE aterotrombótico, e 70\% maior risco de infarto lacunar, o que indica um efeito sinérgico entre esses dois SNPs. A análise do $L D L R$ com base nos haplótipos rs688 e rs5925 realizada em uma população de Taiwan forneceu evidências preliminares que sugerem que polimorfismos genéticos no $L D L R$ estão associados com AVEi (LEE et al., 2012).

\section{Conclusão}

A extensa revisão da literatura realizada no desenvolvimento do presente estudo permite reforçar que a dislipidemia é um dos principais fatores de risco para aterosclerose e DAC e, consequentemente, para o AVEi. A interação entre fatores ambientais e genéticos favorece o desenvolvimento e curso clínico do AVEi. Dentre os fatores genéticos, destacam-se os polimorfismos em genes relacionados com o metabolismo lipídico, tais como as variantes nos genes $L D L R$, apo B, PCSK9, apo E, apo A-I, Apo C-III =, apo $A$-V, CETP e LP. Dentre estes genes, a presente revisão destacou os diferentes polimorfimos no gene $L D L R$ responsáveis pela maioria dos casos de dislipidemias que ocorrem na população em geral. Os polimorfismos no gene $L D L R$ são de extrema importância na etiologia da dislipidemia e aumento do risco para o desenvolvimento da DAC e AVEi.

Devido ao importante papel do polimorfismo no $L D L R$ como causa da dislipidemia, a determinação 
de polimorfismos neste gene em pacientes que sofreram AVEi pode contribuir para identificação de genótipos que estão associados ao maior risco de desenvolvimento de dislipidemia e para a descoberta de novos alvos terapêuticos para o tratamento da dislipidemia nestes indivíduos.

Testes de análise do DNA fornecem um diagnóstico inequívoco e permitem a identificação de indivíduos que carregam uma ou mais variantes genéticas associadas ao risco maior de dislipidemias e, por consequência de AVEi, e assim podem, precocemente, se beneficiarem de medidas preventivas para mudanças de hábitos de vida e se apropriarem de terapias hipolipeminantes que levem em conta o seu perfil genético.

\section{Referências}

ABIFADEL, M.; VARRET, M.; RABES, J. P.; ALLARD, D.; OUGUERRAM, K.; DEVILLERS, M.; CRUAUD, C.; BENJANNET, S.; WICKHAM, L.; ERLICH, D.; DERRE, A.; VILLEGER, L.; FARNIER, M.; BEUCLER, I.; BRUCKERT, E.; CHAMBAZ, J.; CHANU, B.; LECERF, J. M.; LUC, G.; MOULIN, P.; WEISSENBACH, J.; PRAT,A.; KREMPF, M.; JUNIEN, C.; SEIDAH, N. G.; BOILEAU, C. Mutations in PCSK9 cause autosomal dominant hypercholesterolemia. Nature Genetics, New York, v. 34, n. 2, p. 154-156, 2003.

ABRAMCZUK. B.; VILLELA, E. A luta contra oAVBC no Brasil. ComCiência, v. 109, ComCiência[online]. 2009, n.109 [citado 2015-03-16], pp. 0-0 . Disponível em: <http://comciencia.scielo.br/scielo.php?script=sci arttext\&pid=S1519-76542009000500002\&lng-pt\&nrm $=$ iso $>$. ISSN 1519-7654.

ADAMS JUNIOR, H. P.; BENDIXEN, B. H.; KAPPELLE, L.J.;BILLER, J.;LOVE, B. B.;GORDON, D. L.; MARSH, E. E. Classification os subtype of acute ischaemic stroke: definitions for use in a multicenter clinical Trial. TOAST. Stroke, Dallas, v. 24, n. 1, p. 35-41, 1993.

ALMEIDA, E. R.; REICHE, E. M. V.; KALLAUR, A. P.; FLAUZINO, T.; WATANABE, M. A. E. The roles of genetic polymorphisms and human immunodeficiency virus infection in lipid metabolism. Biomed Research International, New York, v. 2013, p. 1-15, 2013.
ANDRADE, F. M.; HUTZ, M. H. O componente genético da determinação dos lipídeos séricos. Ciência \& Saúde Coletiva, Rio de Janeiro, v. 7, n. 1, p. 175$182,2002$.

BARTER, P. J. Hugh sinclair lecture: the regulation and remodeling of HDL by plasma factors. Atherosclerosis, London, v. 3, n. 4, p. 39-47, 2002.

BENLIAN, P.; DE GENNES, J. L.; FOUBERT, L.; ZHANG, H.; GAGNÉ, S. E.; HAYDEN, M. Premature atherosclerosis in patients with familial chylomicronemia caused by mutations in the lipoprotein lipase gene. The New England Journal of Medicine, Waltham, v. 335, n. 12, p. 848-854, 1996.

BERTOLINI, S.; COVIELLO, D. A.; MASTURZO, P.; ZUCCHETTO, E.; ELICIO, N.; BALESTRERI, R.; ORECCHINI, G.; CALANDRA, S.; HUMPHRIES, S. RFLPs of the LDL-Receptor gene: their use in the diagnosis of FH and evaluation of different levels of gene expression on normal subjects. European Journal of Epidemiology, Dordrecht, v. 8, p. 18-25, 1992.

BRANDÃO, A. C.; PINHEIRO JUNIOR, S.; PINHEL, M. A.; ANACLETO, A. M.; GODOY, J. M. P.; GODOY, M. F.; SANTOS, J. E.; SOUZA, D. R. S. Polimorfismo genético da apolipoproteína E na doença arterial periférica. Jornal Vascular Brasileiro, Rio de Janeiro, v. 3, n. 4, p. 317-322, 2004.

BRASIL. Ministério da Saúde. Sistema de Informação de Mortalidade (SIM) e IBGE. [Internet] [acesso em 1 Dezembro 2013]. Disponível em: http:/tabnet. datasus.gov.br/cgi/tabcgi.exe?idb2011/c08.def.

BROWN, M. S.; GOLDSTEIN, J. L. A receptormediated pathway for cholesterol homeostasis. Science, New York, v. 232, n. 4746, p. 34-47, 1986.

CABRAL, N.L. Epidemiologia e impacto da doença cerebrovascular no Brasil e no mundo. Com Ciência, N. 109, P. E5, 2009.

CHATZISTEFANIDIS, D.; GIANNOPOULOS, S.; SPENGOS, K.; VASSILOPOULOU, S.; VEMMOS, K.; DOVA, L.; VARTHOLOMATOS, G.; KYRITSIS, A. P.; GEORGIOU, I.; MARKOULA, S. Apolipoprotein E polymorphisms and ischaemic stroke: a two-center Greek study. European Journal of Neurology, Oxford, v. 21, n. 8, p. 1083-1088, 2014. 
COUTURE, P.; OTVOS, J. D.; CUPPLES, L. A.; LAHOZ, C.; WILSON, P. W.; SCHAEFER, E. J.; ORDOVAS, J. M. Association of the c-514t polymorphism in the hepatic lipase gene with variations in lipoprotein subclass profiles - the Framingham Offspring Study. Arteriosclerosis, Thrombosis, and Vascular Biology, Dallas, v. 20, n. 3, p. 815-822, 2000.

DALLONGEVILLE， J.; MEIRHAEGHE， A.; COTTEL, D.; FRUCHART, J. C.; AMOUYEL, P.; HELBECQUE, N. Gender related association between genetic variations of APOC-III gene and lipid and lipoprotein variables in northern France. Atherosclerosis, London, v. 150, n. 1, p. 149-157, 2000.

DAVIGNON, J.; GREGG, R. E.; SING, C; F. Apoliprotein E polymorphism and atherosclerosis. Arteriosclerosis, Thrombosis, and Vascular Biology, Dallas, v. 8, n. 1, p. 1-21, 1988.

DICHGANS, M. Genetics of ischaemic stroke. The Lancet Neurology, London, v. 6, n. 2, p. 149-61, 2007.

ECKEL, R.H. Lipoprotein lipase: a multifunctional enzyme relevant to common metabolic disease. The New England Journal of Medicine, Waltham, v. 320, n. 16, p. 1060-1068, 1989.

EICHNER, J. E.; DUNN, S. T.; PERVEEN, G.; THOMPSON, D. M.; STEWART, K. E.; STROEHLA, B. C. Apolipoprotein E polymorphism and cardiovascular disease: a HuGE review. American Journal of Epidemiology, Oxford, v. 155, n. 6, p. 487495, 2002.

FALAVIGNA, A.; TELES, A. R.; VELHO, M. C.; VEDANA, V. M.; MAZZOCCHIN, T.; SILVA, R. C.; LUCENA, L. F.; PETRY, F. L.; MOSENA, G.; BRAGA, G. L.; SALVATI, G.; DAHMER, C.; STROPPER, M. O que os pacientes com acidente vascular encefálico sabem sobre sua doença? Revista da AMRIGS, Porto Alegre, v. 53, n. 2, p. 135-138, 2009.

FIEGENBAUM, M.; ANDRADE, F. M.; HUTZ, M. H. Association between plasma lipid parameters and APOC3 genotypes in Brazilian subjects: effect of gender, smoking and APOE genotypes. Clinica Chimica Acta, Philadelphia, v. 380, n. 1-2, p. 175-181, 2007.
FIELDING, C. J.; HAVEL, R. J. Cholesteryl ester transfer protein: friend or foe? The Journal of Clinical Investigation, Michigan, v. 97, n. 12, p. 2687-2688, 1996.

FORTI, N.; SALAZAR, L. A.; DIAMENT, J.; GIANNINI, S. D.; HIRATA, M. H.; HIRATA, R. D. C. Alterações genéticas e colesterolemia: recentes estudos brasileiros. Arquivos Brasileiros de Cardiologia, São Paulo, v. 80, n. 5, p. 565-571, 2003.

GARDEMANN, A.; OHLY, D.; FINK, M.; KATZ, N.; TILLMANNS, H.; HEHRLEIN, F. W.; HABERBOSCH, W. Association of the insertion/ deletion gene polymorphism of the apolipoprotein B signal peptide with myocardial infarction. Atherosclerosis, London, v. 141, n. 1, p. 167-175, 1998.

GRAU, A. J.; BUGGLE, F.; BECHER, H.; WERLE, E.; HACKE, W. The association of leukocyte count, fibrinogen and C-reactive protein with vascular risk factors in ischemic vascular diseases. Thrombosis Research, Rochester, v. 82, n. 3, p. 245-255, 1996.

GO, A.S.; MOZAFFARIAN, D.; ROGER, V.L.; BENJAMIN, E.J.; BERRY, J.D.; BLAHA, M.J.; et al. Heart Disease and Stroke Statistics -2014 Update: A Report from the American Heart Association. Circulation., v.129, p. e28-e292, 2014.

GUDNASON, V.; ZHOU, T.; THORMAR, K.; BAEHRING, S.; COOPER, J.; MILLER, G.; HUMPHRIES, S. E.; SCHUSTER, H. Detection of the low-density lipoprotein receptor gene $P v u$ II intron 15 polymorphism using the polymerase chain reaction: association with plasma lipid traits in healthy men and women. Disease Markers, London, v. 13, n. 4, p. 209220, 1998.

GUZMÁN, E. C. R. et al. Avaliação do efeito dos polimorfismos Hha I da apolipoproteína E e $P v u$ II da lípase lipoprotéica em indivíduos com hipertrigliceridemia. Revista Brasileira de Ciências Farmacêuticas, São Paulo, v. 37, supl. 1, 2001.

HALLMAN, D. M.; BOERWINKLE, E.; SAHA, N.; SANDHOLZER, C.; MENZEL, H. J.; CSÁZÁR, A.; UTERMANN, G. The apolipoprotein E polymorphism: a comparison of allele frequencies and effects in nine populations. The American Journal of Human Genetics, Houston, v. 49, n. 2, p. 338-349, 1991. 
HUMPHRIES, S. E.; KING-UNDERWOOD, L.; GUDNASON, V.; SEED, M.; DELATTRE, S.; CLAVEY, V.; FRUCHART, J. C. Six DNA polymorphisms in the low density lipoprotein receptor gene: their genetic relationship and an example of their use for identifying affected relatives of patients with familial hypercholesterolaemia. Journal of Medical Genetics, London, v. 30, n. 4, p. 273-279, 1993.

JENNER, J. L.; SEMAN, L. J.; MILLAR, J. S.; LAMON-FAVA, S.; WELTY, F. K.; DOLNIKOWSKI, G. G.; MARCOVINA, S. M.; LICHTENSTEIN, A. H.; BARRETT, P. H.; DELUCA, C.; SCHAEFER, E. J. The metabolism of apolipoproteins (a) and B-100 within plasma lipoprotein (a) in human beings. Metabolism, New York, v. 54, n. 3, p. 361-369, 2005.

KUIVENHOVEN, J. A.; JUKEMA, J. W.; ZWINDERMAN,A.H.;DEKNIJFF,P.;MCPHERSON, R.; BRUSCHKE, A. V.; LIE, K. I.; KASTELEIN, J. $\mathrm{J}$. The role of a comum variant of the cholesteryl ester transfer protein gene in the progression of coronary atherosclerosis. The New England Jounal of Medicine, Boston, v. 338, n. 2, p. 86-93, 1998.

LEE, J. D.; LEE, T. H.; KUO, Y. W.; HUANG, Y. C.; HSU, H. L.; LIN, Y. H.; WU, C. Y.; HUANG, Y.C.; LEE, M.; HSIAO, K. M. Polymorphisms at the $L D L R$ locus may be associated with ischemic cerebrovascular disease independent of lipid profile. Current Neurovascular Research, Amsterdam, v. 9, n. 3, p. 200-206, 2012.

LEIGH, S. E. A.; FOSTER, A. H.; WHITTALL, R. A.; HUBBART, C. S.; HUMPHRIES, S. E. Update and analysis of the University College London low density lipoprotein receptor familial hypercholesterolemia database. Annual of Human Genetics, Oxford, v. 72, p. 485-498, 2008.

LEWIS, B. The lipoproteins: predictors, protectors and pathogens. British Medical Journal, London, v. 287, n. 6400, p.1161-1164, 1983.

MARDUEL, M.; CARRIÉ, A.; SASSOLAS, A.; DEVILLERS, M.; CARREAU, V.; DI FILIPPO, M.; ERLICH,D.;ABIFADEL,M.; MARQUES-PINHEIRO, A.; MUNNICH, A.; JUNIEN, C.; FRENCH ADH RESEARCH NETWORK; BOILEAU, C.; VARRET, M.; RABÈS, J. P. Molecular spectrum of autosomal dominant hypercholesterolemia in France. Human Mutation, New York, v. 31, n. 11, p. E1811-E1824, 2010.
MAXWELL, K. N.; FISHER, E. A.; BRESLOW, J. L. Overexpression of PCSK 9 accelerates the degradation of the $L D L R$ in a post-endoplasmic reticulum compartment. Proceedings of the National Academy of Sciences of the United States of America, v. 102, n. 6, p. 2069-2074, 2005.

MCPHEE, S. J.; PAPADAKIS, M. A.; TIERNEY, L. M. Current medical diagnosis \& treatment. 46. ed. New York: McGraw-Hill, 2007.

MENDES-LANA, A. M.; PENA, G. G.; FREITAS, S. N.; LIMA, A. A.; NICOLATO, R. L.; NASCIMENTONETO, R. M.; MACHADO-COELHO, G. L.; FREITAS, R. N. Apolipoprotein E polymorphism in Brazilian dyslipidemic individuals: Ouro Preto study. Brazilian Journal of Medical and Biological Research, São Paulo, v. 40, n. 1, p. 49-56, 2007.

MULDER, M.; LOMBARDI, P.; JANSEN, H.; VAN BERKEL, T. J.; FRANTS, R. R.; HAVEKES, L. M. Low density lipoprotein receptor internalizes LDL and VLDL that are bound to heparan sulphate proteoglycans via lipoprotein lipase. The Journal of Biological Chemistry, Baltimore, v. 268, n. 13, p.9369-9375, 1993.

PATHY, M. S. J.; SINCLAIR, A. J.; MORLEY, J. E. Principles and practice of geriatric medicine. 4. ed. Barcelona: Grafos SA, 2006.

PEACOCK, R. E.; HAMSTEN, A.; JOHANSSON, J.; NILSSON-EHLE, P.; HUMPHRIES, S. E. Associations of genotypes at the apolipoprotein AI-CIII-AIV, apolipoprotein B, and lipoprotein lipase gene loci with coronary atherosclerosis and high density lipoprotein subclasses. Clinical Genetic, Oxford, v. 46, n. 4, p. 273282, 1994.

PEACOCK, R.; DUNNING, A.; HAMSTEN, A.; TORNVALL, P.; HUMPHRIES, S.; TALMUD, P. Apolipoprotein B gene polymorphisms, lipoproteins and coronary atherosclerosis: a study of young myocardial infarction survivors and healthy populationbased individuals. Atherosclerosis, London, v. 92, n. 2-3, p. 151-164, 1992.

PEDERSEN, J. C.; BERG, K. Interaction between low density lipoprotein receptor $(L D L R)$ and apolipoprotein $\mathrm{E}$ (apoE) alleles contributes to normal variation in lipid level. Clinical Genetic, Oxford, v. 35, n. 5, p. 331-337, 1989. 
PEDERSEN, J. C; BERG, K. Normal DNA polymorphism at the low density lipoprotein receptor $(L D L R)$ locus associated with serum cholesterol level. Clinical Genetic, Oxford, v. 34, n. 5, p. 306-312, 1988.

PHAN, T. G.; WRIGHT, P. M.; MARKUS, R.; HOWELLS, D. W.; DAVIS, S. M.; DONNAN, G. A. Salvaging the ischaemic penumbra: more than just reperfusion. Clinical and Experimental Pharmacology and Physiology, Oxford, v. 29, n. 1-2, p. 1-10, 2002.

PIRES, S. L.; RUBEN, J. G.; GORZONI, M. L. Estudo das frequências dos principais fatores de risco para acidente vascular cerebral isquêmico em idosos. Arquivos de Neuro-Psiquiatria, São Paulo, v. 62, n. 3, p. 844-851, 2004.

PORKKA, K. K. V.; TAIMELA, S.; KONTULA, K.; LEHTIMÄKI, T.; AALTO-SETÄLÄ, K.; AKERBLOM, H. K.; VIIKARI, J. S. Variability gene effects of DNA polymorphisms at the apo $B$, apo AI/CIIII and apo E loci on serum lipids: the Cardiovascular Risk in Young Finns Study. Clinical Genetic, Oxford, v. 45, n. 3, p. 113-121, 1994.

ROSAMOND, W.; FLEGAL, K.; FURIE, K.; GO, A.; GREENLUND, K.; HAASE, N.; HAILPERN, S.M.; HO, M.; HOWARD, V.; KISSELA, B.; KITTNER, S.; LLOYD-JONES, D.; MCDERMOTT, M.; MEIGS, J.; MOY, C.; NICHOL, G.; O'DONNELL, C.; ROGER, V.; SORLIE, P.; STEINBERGER, J.; THOM, T.; WILSON, M.; HONG, Y. Heart disease and stroke statistics: 2008 update: a report from the American Heart Association Statistics Committee and Stroke Statistics Subcommittee. Circulation, v.117, n.4, p. e25-146, 2008.

SAKUMA, T.; HIRATA, R. D. C.; HIRATA, M. $\mathrm{H}$. Five polymorphisms in gene canditates for cardiovascular disease in Afro-Brazilian individuals. Journal of Clinical Laboratory Analysis, New York, v. 18, n. 6, p. 309-316, 2004.

SALAZAR, L. A.; CAVALLI, S. A.; HIRATA, M. H.; DIAMENT, J.; FORTI, N.; GIANNINI, S. D.; NAKANDAKARE，E. R.; BERTOLAMI，M. C.; HIRATA, R. D. Polymorphisms of the low-density lipoprotein receptor gene in Brazilian individuals with heterozygous familial hypercholesterolemia. Brazilian Journal of Medical and Biological Research, São Paulo, v. 33, n. 11, p. 1301-1304, 2000a.
SALAZAR, L. A.; HIRATA, M. H.; FORTI, N.; DIAMENT, J.; GIANNINI, S. D.; ISSA, J. S.; HIRATA, R. D. PvuII intron 15 polymorphism at the LDL receptor gene is associated with differences in serum lipid concentrations in subjects with low and high risk for coronary artery disease from Brazil. Clinica Chimica Acta, Philadelphia, v. 293, n. 1-2, p. 75-88, 2000 b.

SALAZAR, L. A.; HIRATA, M. H.; GIANNINI, S. D.; FORTI, N.; DIAMENT, J.; ISSA, J. S.; HIRATA, R. D. Effects of Ava II and Hinc II polymorphisms at the LDL-receptor gene on serum lipid levels of Brazilian individuals with high risk for coronary heart disease. Journal of Clinical Laboratory Analysis, New York, v. 13, n. 6, p. 251-258, 1999.

SALAZAR, L. A.; HIRATA, M. H.; GIANNINI, S. D.; FORTI, N.; DIAMENT, J.; LIMA, T. M.; HIRATA, R. D. Seven DNA polymorphisms at the candidate genes of atherosclerosis in Brazilian women with angiographically documented coronary artery disease. Clinica Chimica Acta, Philadelphia, v. 300, n. 1-2, p. 139-149, 2000c.

SALAZAR, L. A.; HIRATA, M. H.; QUINTÃO, E. C.; HIRATA, R. D. Lipid-lowering response of the HMG-CoA reductase inhibitor fluvastatin is influenced by polymorphisms in the low-density lipoprotein receptor gene in Brazilian patients with primary hypercholesterolemia. Journal of Clinical Laboratory Analysis, New York, v. 14, n. 3, p. 125-131, 2000d.

SANTOS, R. D. (Coord.). III diretrizes brasileiras sobre dislipidemias e diretrizes de prevenção da aterosclerose do departamento de aterosclerose da sociedade brasileira de dislipidemias. Arquivos Brasileiros de Cardiologia, São Paulo, v. 77, supl. 3, p. 1-48, 2001.

SCARTEZINI, M.; HUBBART, C.; WHITTALL, R. A.; COOPER, J. A.; NEIL, A. H.; HUMPHRIES, S. E. The PCSK9 gene R46L variant is associated with lower plasma lipid levels and cardiovascular risk in healthy U.K. men. Clinical Science, London, v. 113, n. 11, p. 435-441, 2007.

SCHUSTER, H.; HUMPHRIES, S.; RAUH, G.; HELD, C.; KELLER, C.; WOLFRAM, G.;ZÖLLNER, N. Association of DNA-haplotypes in the human LDLreceptor gene with normal serum cholesterol levels. Clinical Genetic, Oxford, v. 38, n. 6, p. 401-409, 1990. 
SCHWANKE, C. K.; CRUZ, I. B. M.; LEAL, N. F.; SCHEIBE, R.; MORIGUCHI, Y.; MORIGUCHI, E. H. Análise da associação entre polimorfismo do gene da apolipoproteína $\mathrm{E}$ e fatores de risco cardiovascular em idosos longevos. Arquivos Brasileiros de Cardiologia, São Paulo, v. 78, n. 6, p. 561-570, 2002.

SIEST, G.; PILLOT, T.; RÉGIS-BAILLY, A.; LEININGER-MULLER, B.; STEINMETZ， J.; GALTEAU, M. M.; VISVIKIS, S. Apolipoprotein E: an important gene and protein to follow in laboratory medicine. Clinical Chemistry, Baltimore, v. 41, n. 8, p. 1068-1086, 1995.

STEPANOV, V. A.; PUZYREV, V. P.; KARPOV, R. S.; KUTMIN, A. I. Genetic markers in coronary artery disease in a Russian population. Human Biology, Detroit, v. 70, n. 1, p. 47-57, 1998.

TAYLOR, R.; JEENAH, M.; SEED, M.; HUMPHRIES, S. Four polymorphisms in the LDL receptor gene: their genetic relationship and use in the study of variation at the LDL receptor locus. Journal of Medical Genetics, London, v. 25, N. 10, p. 653-659, 1988.

TESLOVICH, T. M.; MUSUNURU, K.; SMITH,A. V.; EDMONDSON, A. C.; STYLIANOU, I. M.; KOSEKI, M.; PIRRUCCELLO, J. P. Biological, clinical, and population relevance of 95 loci of blood lipids. Nature, London, v. 466, n. 7307, p. 707-713, 2010.

TONK, M.; HAAN, J. A review of genetic causes of ischemic an hemorrhagic stroke. Journal of the Neurological Sciences, Bomova, v. 257, p. 273-279, 2007.

USIFO, E.; , LEIGH, S. E.; WHITTALL, R. A.; LENCH, N.; TAYLOR, A.; YEATS, C.; ORENGO, C. A.; MARTIN, A. C.; CELLI, J.; HUMPHRIES, S. E. Low-density lipoprotein receptor gene familial hypercholesterolemia variant database: update and pathological assessment. Annals of Human Genetics, Oxford, v. 76, n. 5, p. 387-401, 2012.

VILLEGER, L.; ABIFADEL, M.; ALLARD, D.; RABÈS, J. P.; THIART, R.; KOTZE, M. J.; BÉROUD, C.; JUNIEN, C.; BOILEAU, C.; VARRET, M. The UMD-LDR database: additions to the software and 490 new entries to the database. Human Mutation, New York, v. 20, n. 2, p. 81-87, 2002.
WANG, X.; CHENG, S.; BROPHY, V. H.; ERLICH, H. A.; MANNHALTER, C.; BERGER, K.; LALOUSCHEK, W. A meta-analysis of cadidate gene polymorphis,s and ischemic stroke in six study populations: association ot lymphotoxin-alpha in non-hypertensive patients. Stroke, Dallas, v. 40, n. 3, p. 683-695, 2009.

YAMADA, Y.; METOKI, N.; YOSHIDA, H.; SATOH, K.; ICHIHARA, S.; KATO, K.; KAMEYAMA, T.; YOKOI, K.; MATSUO, H.; SEGAWA, T.; WATANABE, S.; NOZAWA, Y. Genetic risk for ischemic and hemorrhagic stroke. Arteriosclerosis, Thrombosis, and Vascular Biology, Dallas, v. 26, n. 8, p. 1920-1925, 2006.

ZHANG, L. F.; YANG, J.; HONG, Z.; YUAN, G.G.; ZHOU, B.-F.; ZHAO, L. G.; HUANG, Y. N.; CHEN, J.; WU, Y.-F. Proportion of different types of stroke in China. Stroke, Dallas, v. 34, p. 20912096, 2003.
Recebido em: 31 jul. 2014. Acesso em:19 nov. 2014. 
